\title{
Sentido de la práctica del Street Workout para los jóvenes escolarizados de la ciudad de Neiva
}

\author{
Jaime Monje Mahecha \\ Universidad Surcolombiana, Colombia \\ jaimemoma@hotmail.com \\ José Hoover Vanegas García \\ Universidad Autónoma de Manizales, Colombia \\ hovg@autonoma.edu.co
}

\section{Resumen}

Este artículo es producto de una investigación realizada con los jóvenes escolarizados que practican Street Workout en la ciudad de Neiva. Los objetivos propuestos para este trabajo fueron comprender el sentido del Street Workout, como actividad saludable y elemento de integración social en relación con la percepción del cuerpo para sus practicantes e interpretar el origen y la finalidad subyacente a su práctica. La investigación tuvo un corte cualitativo con enfoque fenomenológico, respetando la relación que hace la persona de sus propias experiencias, dado que se asume como sinceros las reflexiones expresadas en las entrevistas. Los resultados obtenidos muestran lo que son, lo que hacen, sus aspiraciones deseos, sentimientos y la influencia que esta práctica tiene en la vida de los jóvenes.

Palabras clave: Street Workout, cuerpo, corporeidad, actividad física, motricidad.

\section{Sense of practice of the Street Workout for educated young people in the city of Neiva}

\begin{abstract}
This article is the result of an investigation carried out with the educated young people who practice Street Workout in the city of Neiva. The objectives proposed for this work were to understand the meaning of Street Workout as a healthy activity and element of social integration in relation to the perception of the body for its practitioners and to interpret the origin and purpose underlying their practice. The research had a qualitative cut with a phenomenological approach, respecting the relation that the person makes of his own experiences, given that the reflections expressed in the interviews are taken as sincere. The results obtained show what they are, what they do, their aspirations, desires, feelings and the influence that this practice has on these young people lives.
\end{abstract}

Keywords: Street Workout, body, body, physical activity, motor. 


\section{Introducción}

La investigación buscaba comprender el sentido de una práctica corporal denominada street workout, como actividad saludable y elemento de integración social en relación con la percepción del cuerpo para sus practicantes, jóvenes escolarizados de la ciudad de Neiva, en él se determinan las motivaciones, finalidades de esta actividad que se enmarca dentro del campo de la cultura física.

En la ciudad de Neiva, se han creado escuelas de formación deportiva que utilizan la precaria infraestructura de la ciudad en las diferentes modalidades, dados los triunfos de nuestros representantes a nivel internacional, padres e hijos ven en el deporte una buena opción formativa tanto en la vida cotidiana que como proyecto de vida, y en la medida de sus posibilidades la administración pública respalda este tipo de iniciativas a través de clubes y ligas. Sin embargo dentro del amplio espectro de la cultura física hay otras prácticas como el parkour, el porrismo, y el street workout que van irrumpiendo en la escena urbana, con las cuales se identifican los jóvenes y con las cuales se han ido apropiando de los espacios públicos haciendo gimnasios callejeros.

La irrupción de estas prácticas, se ha dado como respuesta del jóven a la poca cobertura en infraestructura deportiva y a la necesidad de otras alternativas para la ejercitación física, que le permita unas experiencias que enriquezcan su vida social y les brinde la posibilidad de explorar su motricidad, de enriquecer su corporeidad y descubrir otros sentidos, vivencias, que dada su rigidez, probablemente no les brinde el deporte tradicional.

El estudio se realizó con los jovenes que se encuentran en la escolaridad secundaria y universitaria y con profesionales egresados de educación física practicantes del workout de la ciudad de Neiva, la escolaridad es una característica que cambia la percepción de la comunidad sobre los prácticantes de esta manifestación de la cultura física, muchas veces estigmatizados por los elementos de una cultura corporal en la que los jóvenes se reconocen e identifican.

Hay consenso sobre la importancia del ejercicio en los niños y adolescentes en diversos aspectos de su formación. La evidencia científica disponible con respecto al grupo de edades de 5 a 17 años respalda la conclusión de que la actividad física reporta en general beneficios fundamentales para la salud de niños y jóvenes. Esta conclusión está basada en los resultados de varios estudios, que han observado que el aumento de actividad física estaba asociado a unos parámetros de salud más favorables, y de trabajos experimentales que han evidenciado una asociación entre las intervenciones de actividad física y la mejora de los indicadores de salud. Algunos de los beneficios documentados son: mejora de la forma física (tanto de las funciones cardiorrespiratorias como de la fuerza muscular), reducción de la grasa corporal, perfil favorable de riesgo de enfermedades cardiovasculares y metabólicas, mayor salud ósea, y menor presencia de síntomas de depresión (Organización Mundial de la Salud, 2010).

Esfuerzosgubernamentaleshan tenido relativo éxito para ayudar a los jóvenes a descubrir y desarrollar el placer por la actividad física, sin embargo iniciativas como la del workout, en donde de manera autónoma unos jóvenes se reunen y construyen con escasos recursos e infraestructura un medio que los provee de vivencias y experiencias que les permite reflexionar sobre el sentido de sus prácticas, que les permite asumir autonomamente su propio desarrollo como personas, como seres humanos que son responsables de sí mismos, tanto corporal, como socialmente.

Dadas estas aclaraciones, se plantearon preguntas problematizadoras que orientaron la investigación. ¿Cuál es el sentido de la práctica del Street workout, como actividad saludable y elemento de integración social para jóvenes de la ciudad de Neiva en el 2016? ¿Cuáles son las experiencias más significativas de la práctica del Street workout? ¿Cuál es la percepción que los jóvenes tienen de su cuerpo en la practica Street 
workout? ¿Cuáles son los motivos y finalidades que impulsa la práctica del Street workout en los jóvenes?

\section{A que nos referimos cuando aludimos al sentido}

Pensar en el sentido implica trascender lo limtes de los significados y de las explicaciones, ya que las primeras buscan la defición y a descripción de los fenomenos, miestras que la explicación busca las causas de los mismos, busca leyes cientificas o axiomas que le den validez logica a los acontecimientos o fenomenos de la naturaleza. Hablar de sentido implica descifrar las características personales, cuando no individuales que hace que los fenomenos, las vivencias valgan para un ser humano, el sentido busca la finalidad del porque las personas hacen las cosas. El sentido es una categoria ontologica con la cual buscamos profundizar en los anhelos, fantasias y sueños de los jóvenes escolarizados practicantes del street workout en la ciudad de Neiva. El sentido nos permite acercarnos de una manera directa a los seres humanos ya que intenta perpetrar la subjetividad, la intimidad de los pensamientos, las emociones y los sentimientos que hacen de laspersonas seres humanos de conciencia.

Con frecuencia subvaloramos las vivencias o experiencias nuestras al considerarlas comunes $u$ ordinarias, por esta razón rara vez se recuerdan y casi nunca se reflexiona al respecto. Ocasionalmente cuando algo nos remite a ellas las volvemos a recrear, pero sin que seamos reflexivamente conscientes. $\mathrm{Y}$, sin embargo, es importante notar que las experiencias vividas que nunca revisitamos pueden dejar consecuencias latentes y poderosas en nuestro ser presente y nuestro ser futuro. Las experiencias constituyen la fuente del saber y del conocer de los seres humanos, las experiencias, en tanto relación directa de los seres humanos con el mundo son la fuente des ser y del hacer a que toda persona está expuesta en el mundo de la vida.

Para esta investigación el sentido es producto de una incursión en los gimnasios improvisados de los parques públicos de la ciudad de Neiva $\mathrm{y}$, donde los jóvenes decantaron sus vivencias y reflexionaron en torno a la relevancia que ha tenido el street workout en sus vidas, en sus anhelos de configurar su cuerpo y en el descubrimiento de su motricidad, de cómo han asimilado valores y enriquecido su vida social a traves de la convivencia con el grupo.

En la reflexión en busca de sentido, se aprecia una evolución sustantiva dado que reconocen como motivación principal para iniciarse en esta práctica el deseo de configurar un cuerpo fuerte como medio idóneo para poseer el mundo, dado que comienzan testificando a los más avanzados a quienes admiran y su pretensión es tambien ser testificado, sin embargo esta postura inicial cambia, la parte estética cede su importancia por la funcional, el cuerpo fuerte es reemplazado por la riqueza de la motricidad y la corporeidad. En la práctica del Street Workout, los deportistas se descubren a si mismos como sujetos corporales, como sujetos de principios, como sujetos libre, ya que el ejercicio mismo penetra por sus arterias de su propia autonomía, la libertad brota como conciencia corporal en el ejercicio que emerge por su piel y se expresa en sus curpos, en la transformación de su propia carne.

\section{El Street Workout práctica corporal enmarcada en la cultura física}

Dado que no esta reglamentada, ni sus ejercicios sistematizados en un codigo de puntuación, ni tampoco posee una rígida organización, no podemos conceptuar al street workout como un deporte, por lo tanto lo definiremos como una práctica corporal urbana, otra manifestación dentro del amplio repertorio de la cultura física. La cual tiene un alto grado de exigencia física manifestado por la fuerza (especialmente de brazos), para la ejecución de los ejercicios en la barra los cuales pasan de la suspensión al apoyo de diferentes maneras, e incluye elementos de soltar y tomar la barra incluyendo giros.

La ciudad de Neiva cuenta con diferentes escenarios donde se realiza workout, pero ninguno fue diseñado específicamente para esto, por tanto sus practicantes se valen de las porterias en las canchas de fútbol de salón, 
de los pasamanos de los parques infantiles y utilizan infraestructuras similares como barras para desarrollar sus ejercicios, por lo tanto las actividades se dan en un ambiente de informalidad dado que esta practica corporal terminó condicionando estos espacios. La colonización de los espacios, es una consecuencia del workout, pero no en sentido negativa, sino en sentido constructivo, ya que es apropiación, limitaciónn de espacios casi invisibles a la sociedad en general pero que resplandecen a la necesidad de los practicantes de este tipo de actividad.

Este es un ejemplo de cómo estas practicas rebasan previsiones oficiales de desarrollo urbano, que no atienden la emergencia de estos fenómenos citadinos de practicas corporales, terminando los espacios públicos a fuerza de su uso ocupados y apropiados por grupos como el de los jóvenes del estudio. Sin embargo si se hace un análisis de la relación costo beneficio, los jóvenes neivanos mejorarían su calidad de vida con solo un pequeño esfuerzo de la administración municipal, dado que los costos de estas barras son bajos y practicamente no requieren mantenimiento.

El street workout ha crecido alrededor del mundo, en el país y en Neiva, con la ayuda de las redes sociales, especialmente You Tube, que sirvió como medio de difusión de los ejercicios que se iban creando. La sencillez y el poco equipamiento requerido, hacen de esta práctica corporal una buena alternativa para contrarresatar la falta de escenarios deportivos y la drástica reducción de la actividad física de la sociedad actual, especialmente en la población juvenil.

Algunas características claras del Street Workout, son:

Se practica en escenarios públicos.

Generalmente se usa como equipamiento todo tipo de barras, que suelen estar en parques públicos.

Se realizan ejercicios compuestos, siendo los más básicos dominadas, flexiones y fondos.
Se centra en realizar repeticiones de un ejercicio, aunque también hay posiciones estáticas.

Se busca principalmente hacer una demostración de fuerza, aumentando cada vez más la dificultad de los ejercicios.

Se valora una correcta forma en los ejercicios, que dé a entender que se tiene la fuerza suficiente para controlar el movimiento.

Los grupos de practicantes del Street workout no tienen una formalización ante ningún ente estatal o asociado, surgen de manera espontánea mediado por la fraternidad.

\section{Metodología}

Esta investigación cualitativa se desarrolló recurriendo a un enfoque fenomenológico, una de las características de este enfoque "es que respeta plenamente la relación que hace la persona de sus propias vivencias, ya que, al tratarse de algo estrictamente personal no habría ninguna razón externa para pensar que ella no vivió, no sintió o no percibió las cosas como dice que lo hizo". Husserl, citado por (Martinez Migueles, 2006), señala que siempre existe en nosotros un "ambiente de vida presupuesto", un "medio vital cotidiano" y explica "cómo este mundo de vida funge constantemente de trasfondo, cómo sus dimensiones valorativas, prelógicas, son fundacionales para las verdaderas lógicas teóricas", y refiere todo conocimiento teórico a este trasfondo vital natural.

Este método se refiere al camino o actitud para aproximarse al fenómeno de estudio, para que esto se dé, es necesario hacer una reflexión sobre la experiencia desprovista de teorías, polémicas, supuestos y emociones que pueden tergiversarlo, la reflexión sobre la vivencia debe regirse por un lenguaje discursivo y por dispositivos interpretativos sensibles que hacen posible e inteligible el análisis, la explicación y la descripción propias de la fenomenología. La experiencia vivida significa que la fenomenología reflexiona sobre la vida, la existencia humana tal y como se vive.

Los jóvenes participantes de la investigación tuvieron la posibilidad de reflexionar sobre 
sus vivencias y establecer un juicio valorativo de ellas para su vida, descifrando vivencias cotidianas que suscitaron asombro una vez fueron decantadas por sus protagonistas.

En este proceso se siguieron de seis pasos enlazados entre si que es prácticamente imposible separarlos por completo, sin embargo, por constituir actividades mentales diferentes, conviene detenerse en cada una por separado, de acuerdo con la prioridad temporal de la actividad en que ponen énfasis (Martinez Migueles, 2006).

Primer paso: lectura general de la descripción de cada protocolo. En el cual se hizo un esfuerzo para adentrarnos en la realidad ahí expresada y hacerlo de modo más intenso, segundo paso: delimitación de las unidades temáticas naturales. Este paso consistió en pensar acerca del posible significado que pudiera tener una parte en el todo, tercer paso: determinación del tema central que domina cada unidad temática. Se eliminaron las repeticiones y redundancias en cada unidad temática y se determinó el tema central de cada unidad, aclarando y elaborando su significado, cuarto paso: expresión del tema central en lenguaje científico, quinto paso: integración de todos los temas centrales en una estructura particular descriptiva, sexto paso: integración de todas las estructuras particulares en una estructura general. En este paso se integraron en una sola descripción, las estructuras identificadas en los diferentes protocolos, séptimo paso: entrevista final con los sujetos estudiados. En este paso final se hicieron entrevistas con los sujetos participantes en la investigación para darles a conocer los resultados, esto con el fin de considerar datos omitidos, ignorados o, también añadidos.

\section{Resultados}

Para este estudio se contó con la participación de jóvenes en edades comprendidas entre los 16 y 28 años, todos escolarizados, entre media vocacional y universidad, además, hay dos profesionales de la educación física quienes practican street workout desde la secundaria, a partir de sus reflexiones se halló el sentido de sus prácticas. Tras la reflexión fenomenológica se encontraron las siguientes categorías.

\section{El cuerpo}

El cuerpo es para el joven una posesión tangible, fuente de poder y de realización, el cual quiere potenciar, por la vía de la estética, la fuerza y la habilidad, el Street Workout les provee esta posibilidad. A diferencia de las posturas mecanicistas que consideran el cuerpo como un producto de la naturaleza, en la fenomenología, el cuerpo es una unidad de significación, ya que la única manera de conocer el cuerpo es vivirlo, experimentarlo y la experiencia que tenemos del cuerpo propio se distiende hacia un mundo significativo. Las percepciones hacen parte de la 'corriente' de las vivencias, de hecho, son expresiones o relaciones de sentido que establece mi cuerpo en el mundo. "El cuerpo-propio no es el objeto del mundo, sino el medio de nuestra comunicación con él al mundo; es el horizonte latente de nuestra experiencia" Gallo, Dadavid., 2006).

Esta corporeidad se afianza después de una primera fase en la que predomina lo netamente físico, por afianzar una imagen de virilidad, estética y desarrollo corporal. "Claro, el desarrollo muscular es evidente, a mí me sacaron del bajo mundo, inicie el Street Workout pero yo llevaba sin hacer ejercicio como un año y ya tenía barriguita, y esa fue una de las cosas que me motivo a volver a la actividad física y el cambio fue del cielo a la tierra, ya en dos meses volvió a verse el cambio en mi cuerpo". Se pondera mucho las bondades del cuerpo, no solo del cuerpo joven y fuerte y esto tiene mucho sentido, nuestro cuerpo es nuestra carta de presentación, es una mediación de nuestra existencia en el mundo, no debemos olvidar que a través de él accedemos y formamos parte de éste, para Merleau Ponty el cuerpo es nuestro anclaje en un mundo. (Merleau-Ponty, 1975).

Encontrar ese cuerpo que han idealizado, implica dos aspectos íntimamente ligados, el placer y el dolor en una intima dicotomía, se crea la convicción de que es necesario el trabajo y la disciplina para alcanzar algún propósito 
en la vida. Según Nietzsche, poner en juego nuestras capacidades contra retos adecuados, autoimponernos dificultades y desafíos que nos obliguen a trabajar a fondo nuestras condiciones físicas y mentales es sinónimo de experimentar la felicidad. Turró, cita a Platón con la siguiente sentencia. ¿No hacen lujuriosos a los jóvenes e incapaces de trabajar con el cuerpo ni con el alma, y perezosos y demasiado blandos para resistir el placer o soportar el dolor? (Mora, 2007).

De la concepción estética cambia por la idea de un cuerpo funcional, no basta con adquirir capacidades físicas ni con el aumento del volumen, ahora pretenden el desarrollo de la corporeidad, de su motricidad. Apreciamos una evolución conceptual, con una apropiación sobre los alcances del cuerpo y su desarrollo motriz. El cuerpo reivindicado como medio de expresión. Aparece aquí el moverse como aquel movimiento que deja de ser simplemente un cambio de lugar, por un movimiento que entra en relación con el mundo de la vida, como aquel movimiento que está ligado a la vivencia, las emociones, las percepciones, el estado de ánimo, el sentir corporal, a la conciencia encarnada, es decir, es la motricidad misma (Manen Max, 2016).

La búsqueda de esa corporeidad genera otras inquietudes, además de la actividad física como dimensión de salud, indagan sobre alimentación y tratan de hacerlo de manera saludable, y por supuesto, no consumen sustancias antinaturales en busca de rendimiento, dado que el fin preponderante no es el triunfo porque no hay una sobrevaloración de la competencia, esta práctica tiene un carácter más libre, espontáneo y voluntario, se puede apreciar que antes que vencer al adversario o conseguir un record, lo que se busca es la solidaridad, el crecimiento personal y colectivo.

\section{Testificación, cooperación}

Ver a otros jóvenes en los parques de su vecindario o en videos por you tube, los motivo para iniciarse en el workout, en primer lugar se sorprendieron con la fortaleza de los ejecutantes, es decir hubo reconocimiento del cuerpo del otro, lo cual fue ratificado al ver el amplio número de elementos que ejecutaban, sus pares se constituyeron en referentes, por lo que quisieron emularlos.

Una siguiente fase identificada fue cuando mejoraron su condición física y técnica y con esto el dominio de elementos cada más complejos, ahora comenzaban a reconocer su cuerpo, a admirarlo, una vez seguros de su progreso, se da una tercera fase cuando tienen sus propios admiradores, es el reconocimiento que hace el otro a su cuerpo, esta testificación por parte de los otros es una recompensa al logro de habilidades y desarrollo corporal, lo que les eleva la autoestima y les da confianza para asumir liderazgos. La riqueza tangible, evidente y más próxima que percibe el joven es su cuerpo, Bernard lo expone de la siguiente manera: el cuerpo es "la principal estructuración simbólica del hombre, y su esencia y existencia constituyen la única posibilidad de permanencia en el mundo y trasciende el determinismo biológico para convertirse en integrador de las dimensiones del ser humano" (Bernard M, 1980).

Los iniciados pronto encuentran en esta práctica una conjunción de elementos que satisfacen sus demandas de actividad física, comunicación, recreación, juego, aprendizaje, los jóvenes encontraron en esta actividad, unas características diferenciadoras en la interacción con el grupo, muchos vienen de deportes tradicionales y aprecian las diferencias, ven que la competencia es reemplazada por los logros personales pero en este camino se encuentran con la solidaridad del grupo, es decir, es tan satisfactorio la adquisición de un nuevo elemento tanto para el ejecutante como para sus compañeros. "Claro, tanto lo que yo logro como lo que logran mis compañeros es gratificante, levanta el ánimo, la moral a nivel personal y colectivo... Lo que más me llamó la atención de esta prácticaes que uno conoce gente "Admirable" que tiene muy buena disciplina, tienen un buen nivel gimnástico y muy buena condición física. Se conoce gente buena y humilde, se comparte buenos momentos, es como una familia. Bueno. Además, esta es una disciplina alternativa, que no se necesita de mucho, en mi caso aprendí solo en una cancha de futbol, quisiera destacar esto". 


\section{Tiempo libre, creatividad y espacio público}

Las autoridades educativas y de salud propenden por que los jóvenes den buen uso al tiempo libre, argumentando evitar malas influencias y contrarrestar el sedentarismo, este anhelo gubernamental y educativo no siempre es exitoso, sin embargo el sentido que tiene esta experiencia para estos jóvenes practicantes de workout hay que apreciarlo con más atención.

Están organizados de tal manera que sus pares son sus profesores, a través del ensayo y el error van mejorando la condición física predisponiéndolos física y psicológicamente para asimilar y ejecutar nuevos elementos, esto es una muestra de emprendimiento, pero también de sensibilización social y solidaridad.

El sentido de la creatividad e innovación viene de los retos motrices, una vez dominado un ejercicio, intentan otro, y van ampliando la dificultad de los mismos, lo que los obliga a indagar por la mejor técnica antes de acometerlos. "Si, yo llegue con influencia de gimnasia, de porras, que ayudan, pero aquí es una mezcla de todo, se requiere de fuerza, de coordinación, uno aquí saca nuevas técnicas para nuevos elementos, detallitos pequeños que van sumando a nuevos ejercicios....o sea logré cosas que antes no pensé que podía hacer, y no imaginé que podía hacer y lo logré hacer".

Pero estos logros no sólo tienen un valor específico, sino que se hacen en espacios públicos que han ocupado, adaptado y apropiado como gimnasios callejeros, con este empoderamiento se han hecho a un territorio, ahora cuentan con un punto de encuentro. Esto pone en evidencia que los jóvenes demandan practicas corporales urbanas que no tienen eco en la administración pública, el desarrollo urbano no atiende este tipo de requerimientos ni en espacios ni en implementos.

Esta práctica urbana de los jóvenes en los parques y espacios públicos es informal, no institucionalizada pero no clandestina, hay que destacar frecuentes casos de acompañamiento familiar, los padres asisten a los festivales para apoyar a sus hijos, pero también a las prácticas por lo que se ha iniciado una difusión a otros miembros del entorno familiar.

En el Esquema No. 1 ilustra las diversas categorías encontradas que le dan sentido a la práctica del street workout a los jóvenes escolarizados de la ciudad de Neiva.

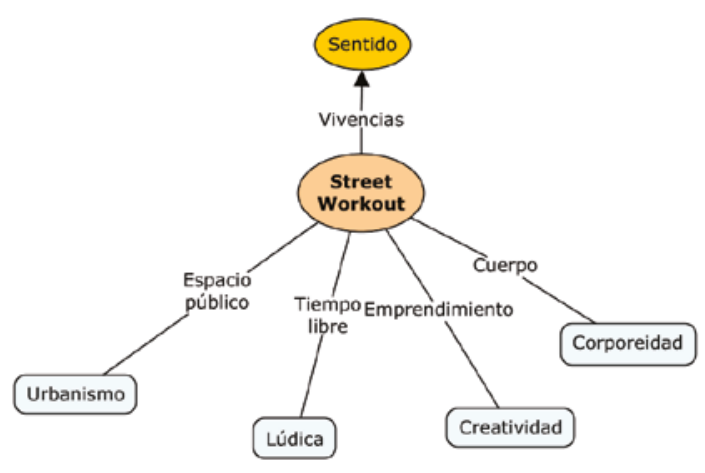

Esquema 1. Categorías de sentido de la practica del street workout en los jóvenes escolarizados de la ciudad de Neiva.

\section{Discusión}

Enmarcar la juventud como grupo etario, es un esfuerzo complejo, según Freixa, la aparición de lo juvenil se da con los denominados muchachos en la sociedad industrial, el surgimiento de esta categoría se asocia a transformaciones que sufrieron instituciones sociales como la familia, la iglesia, el ejército, la escuela (Freixa, 1984).

La segunda revolución industrial introdujo avances tecnológicos que exigían de niños y jóvenes una cierta preparación, lo que los alejó del mundo laboral expulsándolos a la escuela o a la calle. Mientras que para los burgueses la juventud representaba un periodo de moratoria social marcada por el aprendizaje escolar y el ocio forzoso. Es por esta razón que la categoría joven, se asume como una construcción social de algo que se llama escolaridad, de aquel que puede acceder a la moratoria o sea del joven con ciertas condiciones de vida, joven varón ya que las jóvenes entraron en esta moratoria muy tardíamente. 
Los jóvenes de esta investigación tienen el "privilegio" de estar en esta moratoria, y disponer de ese ocio forzoso, en espera de entrar a los tradicionales círculos de producción una vez sean considerados calificados para esta transición dado que están inmersos en el sistema escolar. Justamente han hecho del street workout, una especie de satisfactor de ese ocio forzoso, lo han utilizado como un ocio creativo en la experimentación con su cuerpo. $\mathrm{Al}$ respecto Merleau-Ponty afirma, desde la fenomenología de la 'existencia corpórea', el cuerpo es el 'medio' de nuestro 'ser-hacia-el-mundo (Gallo, Cadavid, 2006).

Estamos frente a una experiencia educativa, no solo en términos de la cultura física sino tambien de la convivencia y la formación ciudadana utilizando como medio una práctica corporal urbana tadicionalmente estigmatizada, nos preguntamos ¿Qué hace diferente esta práctica para que los jóvenes en sus reflexiones no hayan citado la estigmatización? Creemos que la diferencia justamente que estos actores estan escolarizados, e inclusive algunos profesionales de la educación física.

El hecho de que seán jóvenes que conviven con sus familias, que asisten con regularidad al colegio o a la universidad, denota que mantienen una disciplina académica que no riñe con esta práctica, pero que además cuentan con el apoyo familiar y un elemento muy diferenciador es que no son consumidores.

En esos espacios se tienen vivencias y experiencias que desbordan marcos preexistentes, permitiendo la irrupción de otras alternativas, esos espacios posibilitan la realización de prácticas corpóreas, que permiten descubrir potencialidades en la medida que se asumen nuevos retos motrices, hay una toma de conciencia de atributos que se ignoraban o subvaloraban y por tanto yacían inutilizados tanto a nivel personal como colectivo. García, en Maurice Merleau-Ponty. Filosofía, corporalidad y percepción, cita a $\mathrm{D}$. Le Breton, quien relata una experiencia, "Como han advertido los antropólogos, no se trata aquí de meras metáforas sino de una identidad sustancial percibida $y$ experimentada entre lo vegetal y el cuerpo. En esta experiencia melanesia del cuerpo se muestra la misma ausencia de individuación que se observaba en los Dogon: el hombre sólo existe por su relación con el otro y la naturaleza, y el cuerpo no es el soporte de una individualidad" (García, E, 2012).

La responsabilidad sobre la actividad física se ha asignado a la escuela, esta institución ha delegado tal propósito al área de educación física. Con la creación de Coldeportes a través del decreto 2743 de 1968, la presentación del plan de estudios de los colegios INEM por el decreto 363 de 1970 y la reglamentación de los juegos interescolares e intercolegiados en 1971, la Educación Física se ha valido del deporte como medio para fomentar la práctica de la actividad física, esto se manifiesta en el contenido de los programas basados en fundamento $\mathrm{y}$ perfeccionamiento técnico en los deportes. (Ministerio de Educación Nacional, 2015).

Por ese motivo la Educación Física en la institución educativa ha privilegiado la práctica deportiva, se pretende detectar los posibles talentos y futuras figuras de diferentes modalidades deportivas. Esta es la razón por la cual el colegio promueve la competencia deportiva como representación.

Tal postura tiene muchos simpatizantes y parece haber logrado su cometido dados los triunfos de los deportistas colombianos a nivel internacional en diversas modalidades deportivas, sin embargo no todos nuestros deportistas están escolarizados y hay muchos estudiantes que no reconocen al deporte como único medio para promover la actividad física, los intereses de estos últimos son llenados en otros espacios desarrollando nuevas creaciones en el campo de la cultura física, como el Parkour, el porrismo o el Street Workout.

El joven a través de estas experiencias resuelve situaciones que no han sido contempladas en la escolaridad tradicional, muchas veces el estudiante pasivo de la clase de educación física se reinventa en estos escenarios urbanos, muchos de ellos han sido llamados para integrar 
selecciones representativas del colegio, pero prefieren continuar con su grupo haciendo workout, dado el valor agregado que perciben y la satisfacción que les genera.

Núñez LJ, en una investigación sobre el Auto concepto físico y satisfacción con la vida en practicantes de ejercicio físico, cuyo objetivo fue analizar las relaciones entre la motivación intrínseca, el auto concepto físico y la satisfacción con la vida en practicantes de ejercicio físico, encontraron que hombres y mujeres que practican ejercicio físico sin presiones externas y porque les gusta, están más satisfechas con sus vidas y esto, en parte, se debe a que se perciben en mejor forma a nivel físico (Nuñez, LJ, 2012). Observaron en el contexto de la actividad física que la motivación intrínseca predice el auto concepto físico, entendido como la percepción que una persona tiene de sus factores físicos, que forma a partir de las experiencias y las relaciones con el medio. Esta apreciación coincide con el sentido encontrado por los jóvenes en la investigación, las gratificaciones que les genera el workout, se refleja en otras dimensiones de sus vidas.

Dado el gran beneficio que reporta este tipo de prácticas, manifestado en la buena utilización del tiempo libre, en la actividad física a estas edades, al enriquecimiento de su vida social, al despertar de la cooperación y solidaridad, al buen uso de los espacios públicos, a la formación ciudadana, pero además de los bajos costos en escenarios e implementación, se debe atender de manera prioritaria estas prácticas corporales dentro de los planes de desarrollo urbano.

El desarrollo de esta investigación con jóvenes escolarizados, abre la oportunidad para indagar sobre los grupos practicantes de street workout, que no son escolarizados, percibidos por la comunidad como vagos y consumidores, es posible que tambien en el grupo sean amigables, abiertos y solidarios para compartir los secretos de los ejercicios y que a pesar de la rotulación sean disciplinados. Este sería un interesante tema para abordar en el futuro.

\section{Conclusiones}

El sentido de la práctica del Street workout para el joven se da al tomar conciencia de su cuerpo, al descubrir su corporeidad expresada en la interacción social con sus pares y en la responsabilidad de su cuidado.

La carencia de un sitio para la actividad física, posibilitó al joven reclamar el espacio público comounlugarno solode práctica sinocomo punto de encuentro y una oportunidad para socializar. En este sentido los pasamanos del parque, las porterías de microfútbol, son reclamados por los jóvenes como su territorio, este paisaje urbano lo utilizan con responsabilidad y autonomía.

En el contexto de la práctica, se da la inclusión, lo cual posibilita un ambiente de confianza y familiaridad donde los jóvenes exponen sus aspiraciones, deseos y sentimientos de manera sincera. Este elemento puede explicar el potencial socializador del Street Workout.

El cuerpo del joven es percibido como fuente de creación y es valorado por sus posibilidades funcionales. En este sentido se aprecia una evolución del concepto que trasciende lo estético.

La pretensión inicial del joven es construir un cuerpo fuerte y armónico, sin embargo, se da cuenta que el Street workout es un movimiento que le posibilita experiencias y emociones, a partir de estas vivencias adquieren otro sentido el cual es enriquecer su motricidad.

Los hábitos, las actitudes, las conductas y las decisiones de los jóvenes practicantes del Street workout, ponen de presente la construcción de un estilo de vida saludable.

Un elemento formativo derivado de la práctica del Street workout, es la idea de que para alcanzar una meta hay que trabajar y perseverar, por lo que se debe tener voluntad y convicción, para sobreponerse a la fatiga y al dolor, sensaciones que son compensadas con gratificaciones que derivan en placer. 
Entre las diversas categorías encontradas, se puede apreciar nexos evidentes, sin embargo, la categoría cuerpo emerge con más fuerza, al punto de posicionarse como un eje alrededor del cual gravitan las demás.

Entre los jóvenes practicantes del Street workout hay una configuración identitaria, que parte del reconocimiento del propio cuerpo, que reconoce el cuerpo del otro, pero que también requiere de una testificación plasmada en el reconocimiento que le hace el otro a su cuerpo.

\section{Referencias bibliográficas}

Bernard M. (1980). El cuerpo. Buenos Aires: Paidós.

Freixa C. (1984). De jóvenes, bandas y tribus. Antropología de la juventud. Barcelona: Ariel.

Gallo, L. E. (2006). El ser-corporal-en-el-mundo como punto de partida en la fenomenología de la existencia corpórea. Pensamiento Educativo. Vol. 38, pp. 46-61.

García E. (2012). Maurice Merleau-Ponty. Filosofía, corporalidad y percepción. Buenos Aires: Rhesis.

Manen M. v. (2016). Fenomenología de la práctica. Métodos y donación de sentido en la investigación y la escritura fenomenológica. Popayán: Editorial Universidad del Cauca.

Martínez Migueles, M. (2006). Ciencia y arte en la metodología cualitativa. México, D.F.: Trillas.

Merleau-Ponty M. (1993). Fenomenología de la percepción. Madrid: Editorial Planeta-De Agostini.

Ministerio de Educación Nacional Colombia. Lineamientos curriculares de la Educación Física. www.mineducacion.gov.co. Último acceso el 16 de Febrero de 2015, de http://www.mineducacion.gov.co/1621/ articles-339975_recurso_10.pdf.

Mora F. (2007). Neurocultura. Madrid: Alianza Editorial.

Núñez, LJ. (2012). Motivación intrínseca, autoconcepto físico y satisfacción con la vida en practicantes de ejercicio físico. Revista Iberoamericana de Psicología del Ejercicio y el Deporte 8 (1), 39-58.

Organización Mundial de la Salud. (2010). Recomendaciones mundiales sobre actividad física para la salud. Ginebra, Suiza: blossoming.it. 